\title{
Intolerancia gastrointestinal a metformina y diverticulitis aguda
}

\author{
Carlos Walter Contreras-Camarena' ${ }^{1}$, Humberto Lira-Veliz ${ }^{2}$ \\ 'Servicio de Medicina Interna. Hospital Nacional Dos de Mayo. Universidad Nacional Mayor de San Marcos. Lima. Perú \\ ${ }^{2}$ Departamento de Emergencias y Cuidados Críticos. Hospital Nacional Dos de Mayo. Universidad Nacional Mayor de San Marcos. Lima. Perú
}

Recibido: 18/09/2020

Aceptado: 23/11/2020

En línea: 31/12/2020

Citar como: Contreras-Camarena CW, Lira-Veliz H. Intolerancia gastrointestinal a metformina y diverticulitis aguda. Rev Esp Casos Clin Med Intern (RECCMI). 2020 (Dic); 5(3): 123-125. doi: 10.32818/reccmi.a5n3a9.

Cite this as: Contreras Camarena CW, Lira-Veliz H. Gastrointentinal intolerance metformin and acute diverticulitis. Rev Esp Casos Clin Med Intern (RECCMI). 2020 (Dec); 5(3): 123-125. doi: 10.32818/reccmi.a5n3a9.

Autor para correspondencia: Carlos Walter Contreras-Camarena. ccontrerashndm@hotmail.com

\begin{tabular}{l} 
Palabras clave \\
\hline$\triangleright$ Metformina \\
$\triangleright$ Efectos adversos \\
$\triangleright$ Diarrea \\
$\triangleright$ Diverticulitis colónica
\end{tabular}

\section{Keywords}

$\triangleright$ Metformin

$\triangleright$ Adverse effects

$\triangleright$ Diarrhea

$\triangleright$ Colonic diverticulitis

\begin{abstract}
Resumen
La intolerancia a metformina se presenta en el 20 a 30\% de los diabéticos tratados con este fármaco; se suspende definitivamente en el $5 \%$ por efectos gastrointestinales severos. Presentamos el caso de un varón, de 65 años, diabético 2, con enfermedad diverticular, que presentó diarreas y dolor abdominal reiteradamente por metformina. Fue admitido en la unidad de cuidados intensivos por diverticulitis aguda y dado de alta con insulina y suspensión definitiva de metformina. Sugerimos, considerar la probabilidad de incremento del riesgo para intolerancia a metformina en presencia de alteraciones morfológicas intestinales.
\end{abstract}

Abstract
Metformin intolerance occurs in 20 to $30 \%$ of diabetics; it is definitively suspended in 5\% due to severe gastrointestinal
effects. We present the case of a 65 -year-old male, diabetic type 2, with diverticular disease, who repeatedly presented
diarrhea and abdominal pain due to metformin. He was admitted to the intensive care unit for acute diverticulitis and
discharged with insulin and definitive withdrawal of metformin. We suggest considering the probability of increased risk
for metformin intolerance in the presence of intestinal morphological changes.

\section{Puntos destacados}

$\triangleright$ Se debe sospechar intolerancia a la metformina en todo diabético que presenta síntomas gastrointestinales agudos en las 24-72 horas posteriores a su administración oral.

$\triangleright$ El síntoma cardinal en este caso fue la diarrea, probablemente, por coexistencia de enfermedad diverticular colónica.

\section{Introducción}

La metformina es el medicamento de elección en diabéticos tipo 2. Su intolerancia se caracteriza por dolor abdominal, náuseas y diarreas entre uno a tres días después de haber iniciado el tratamiento, afectando la calidad de vida y adherencia al tratamiento. Se reporta en el 30\% de los pacientes, usualmente, desparece al suspender o reducir la dosis, pero puede persistir y motivar su retiro definitivo en el $5 \%$ de los casos $^{1,2}$.

Dujic et al. ${ }^{3}$ postulan que la intolerancia a la metformina está relacionada con la disfunción del sistema de transporte de cationes orgánicos (TCO), en la superficie del enterocito, que se encargan de transportar el fármaco desde el lumen hasta la circulación sistémica. Reportaron intolerancia a la metformina 2 a 4 veces más en diabéticos con inhibición farmacológica de los TCO'.
Elbere et al. ${ }^{4}$ reportaron modificación del microbiota intestinal, incremento relativo de patógenos oportunistas Escherichia-Shigella spp. y disminución significativa en la población bacteriana, específicamente de las familias Peptostreptococcaceae y Clostridioides.

Los hallazgos de McCreight et al. ${ }^{5}$ apoyan la hipótesis de que las alteraciones morfológicas intestinales podrían desempeñar un papel importante en la intolerancia a la metformina. No encontraron diferencias significativas en la farmacocinética de metformina entre pacientes diabéticos tolerantes e intolerantes, sugiriendo la asociación de intolerancia con alteraciones morfológicas en el lumen o en el enterocito.

\section{Caso clínico}

\section{Antecedentes personales}

Varón de 65 años, enfermero, no fumador, diabético tipo 2. Desde hace tres años está en tratamiento irregular con metformina por molestias gastrointestinales, severas entre tres y cuatro días después de su administración. Como antecedente está diagnosticado de enfermedad diverticular colónica desde hace cinco años (Figura 1). 


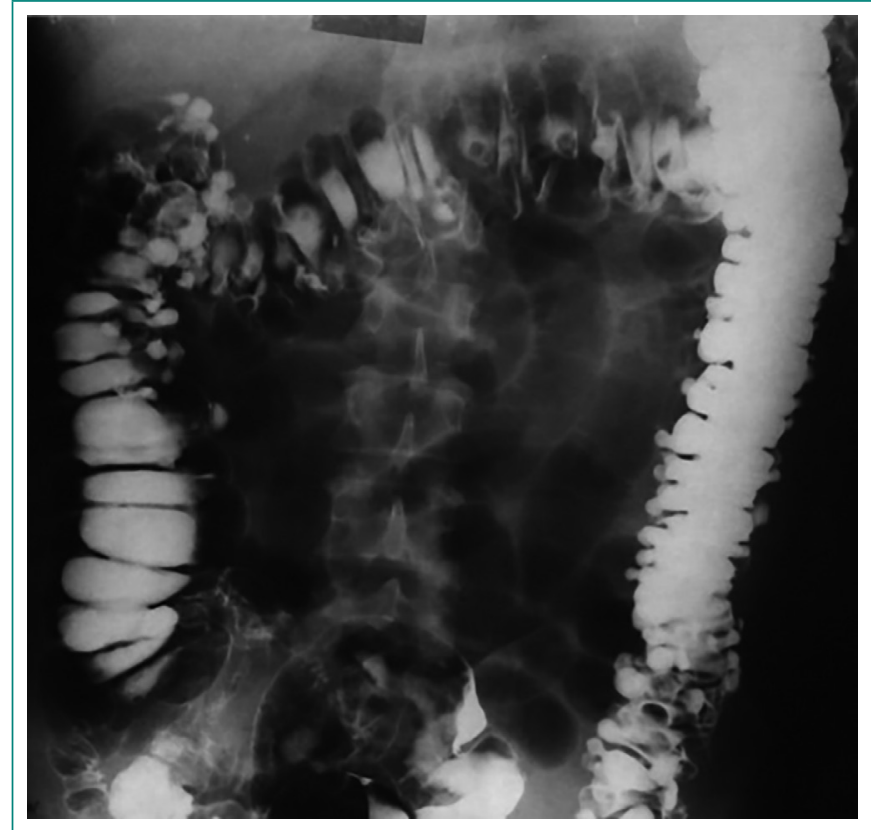

Figura 1. Imágenes saculares de adición que compromete gran extensión del marco colónico.

\section{Enfermedad actual}

Acudió a Urgencias por diarreas acuosas agudas con deshidratación posterior a la ingesta de metformina $850 \mathrm{mg}$ cada 12 horas. Se suspendió el tratamiento y con la mejoría se le dio el alta con la misma prescripción, dieta y ejercicios, basados en sus niveles de glicemia en ayunas de $158 \mathrm{mg} / \mathrm{dL}$ y HbA1c: $8,5 \%$.

Al continuar con deposiciones líquidas con la administración de metformina, se suspendió el tratamiento y se consultó con otro médico. Pasó a tratamiento ambulatorio con metformina, con la posibilidad de que sus síntomas mejorasen en una o dos semanas. El paciente mantiene el tratamiento con tabletas $850 \mathrm{mg} / 24 \mathrm{~h}$, a pesar de la diarrea y el dolor abdominal recurrente.

Dos días antes de ingresar en Urgencias incrementa la frecuencia de diarreas (3 a 5 deposiciones por día), automedicándose con tabletas de hioscina. Cuatro horas antes de su ingreso en Urgencias, presentó incremento de dolor abdominal cólico, diarreas acuosas, fiebre y compromiso del estado general. Entró en la unidad de cuidados intensivos (UCI) con sospecha de sepsis abdominal y diverticulitis aguda.

\section{Exploración física}

- Examen clínico: PA: 95/47 mmHg, FC: 114 lpm, FR: 20 rpm. T: 38,5 ㄷ. IMC: $21,72 \mathrm{Kg} / \mathrm{m}^{2}$. Talla: $1,77 \mathrm{~m}$. Peso: $68 \mathrm{Kg}$.

- Adelgazado, longilíneo en mal estado de hidratación.

- Cardiovascular: ruidos cardíacos, rítmicos, taquicárdicos.

- Pulmones: sin alteraciones.

- Abdomen: dolor en flanco y fosa iliaca izquierda a la palpación profunda.

- Ruidos hidroaereos incrementados, ausencia de visceromegalia.

- Examen neurológico: normal.

\section{Pruebas complementarias}

- Glucosa 156 mg/dL, HbA1c: 8,5\%.

- Urea $16 \mathrm{mg} / \mathrm{dL}$, creatinina $1,25 \mathrm{mg} / \mathrm{dL}, \mathrm{BUN}$ : $19 \mathrm{mg} / \mathrm{dL}$.

- Na: $138 \mathrm{mEq} / \mathrm{L}$. K: 3,7 mEq/L. Cl: $105 \mathrm{mEq} / \mathrm{L}$.

- Gasometría: PH: 7,34. HCO3: 19 mmol/L, PaCO2: 41 mmHg, PaO2: 91 mmHg
- Anión Gap: 18,7 mEq/L Hb:14,6 gr/dL, Hcto: 44.4\%, VCM: 90 fL.

- HCM: 29,4 pg. CHCM: 32,7gr/dL. RDW: 13,7.

- Leucocitos: 13,9 $\times 10^{3} / \mathrm{LL}$. Segmentados: $88 \%$, linfocitos: $26 \%$, monocitos: $6 \%$, eosinófilos: $2 \%$, basófilos: $0 \%$. Abastonados: 6\%

- Plaquetas: $152 \times 10^{3} / \mathrm{LL}$. LDH:131 U/L (50-150).

- PCR 58,4 mg/L (0-10).

- Pro-Calcitonina (PCT): 2,03 ngm/mL $(<0,5)$.

- Ultrasonografía: Engrosamiento parietal irregular de colon sigmoide de hasta $6 \mathrm{~mm}$, hasta una extensión longitudinal de $63 \mathrm{~mm}$. Imágenes diverticulares en colon descendente con reborde hipoecogénico.

- TAC abdomino-pélvico contrastada: engrosamiento de la pared diverticular (6 mm) a lo largo de la unión del colon descendente y sigmoide, sugestivo de diverticulitis aguda leve del colon descendente distal. (Figura 2).

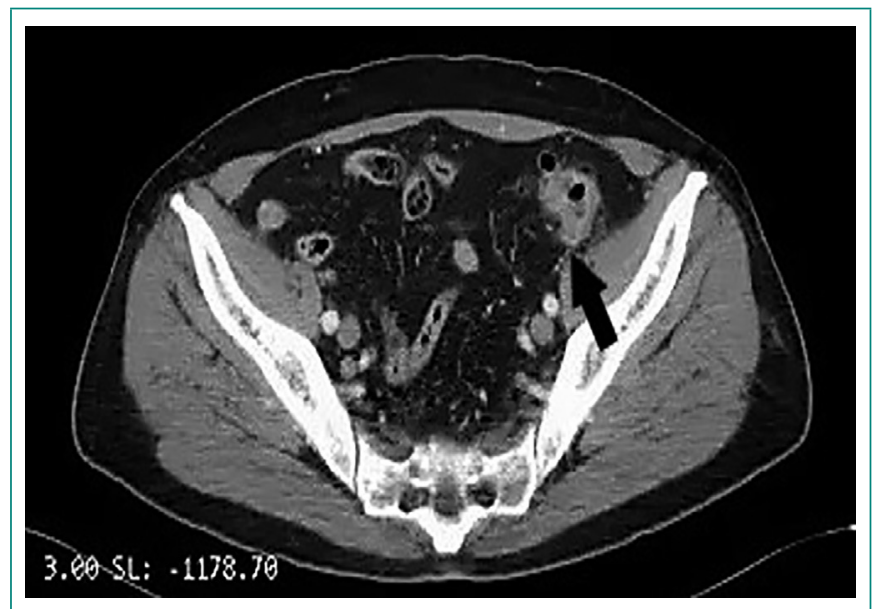

Figura 2. Engrosamiento diverticular posterior en la unión del colon descendente y sigmoide, compatible con diverticulitis leve.

\section{Evolución}

En la UCl recibió soporte hemodinámico, coloides y cristaloides, ciprofloxacino 200 EV mg c/12 horas, metronidazol 500 mg EV c/8 horas y hioscina $50 \mathrm{mg}$ EV c/8 horas.

Se logró mejoría clínica al cuarto día de evolución, con la normalización gasométrica y del hemograma al quinto día. Se le dio el alta con insulinoterapia y suspensión definitiva de metformina.

\section{Diagnóstico}

Por la secuencia semiológica, antecedente de molestias gastrointestinales reiterativas, entre 24 a 48 horas posterior a la administración de metformina, y por franca mejoría al suspenderla, se concluyó: intolerancia a metformina.

Por el cuadro clínico, fiebre, taquicardia, dolor abdominal, leucocitosis con neutrofilia, marcadores de sepsis elevados e imágenes tomográficas, se confirmó: diverticulitis aguda con sepsis severa

\section{Discusión y conclusión}

Modelos experimentales encontraron transporte deficiente a través de la membrana epitelial intestinal, produciendo un incremento de la concentración de metformina y elevando las concentraciones de lactato dentro del enterocito. Además, la metformina modifica la secreción de GLP-1 y altera el 
microbioma, esta última ha sido descrito como un mecanismo de acción adicional del medicamento ${ }^{6}$.

Por otro lado, se ha sugerido una predisposición genética en pacientes intolerantes a metformina específicamente en aquellos genotipos SLC22A1 que expresan actividad incompleta de los transportadores de cationes orgánicos (TCO), tanto en el borde apical y basolateral del enterocito, promoviendo el incremento de la concentración intraluminal de metformina y duplicando el riesgo de intolerancia a metformina ${ }^{6,7}$.

La aparición precoz de la diarrea inmediatamente después de la administración de metformina y su mejoría después de retirarla, así como los antecedentes de cuadros similares previos, determinaron el diagnóstico de intolerancia a la metformina. Los efectos adversos gastrointestinales de metformina no solo estarían relacionados con un incremento de metformina luminal y epitelial sino que, además, con un incremento del péptido intestinal vasoactivo, interacción con el sistema serotoninérgico intestinal y modificación en la secreción de ghrelina.

Existen estudios experimentales utilizando transferencia fecal de pacientes diabéticos en tratamiento con metformina a ratones de laboratorio que han encontrado mejoría en la tolerancia a la glucosa en animales de experimentación. Estos resultados sugieren la existencia de un mecanismo hipoglucémico adicional de metformina por alteración del microbiota ${ }^{8}$.

Estudios recientes encontraron incidencia de diverticulitis menor al 5\% en pacientes con enfermedad diverticular colónica, reportándose mayor incidencia en obesos y a edad relativamente menor ${ }^{9}$. En el caso presentado, el paciente carece de factores que incrementan el riesgo para desarrollar diverticulitis (IMC $\geq 30$, tabaquismo, administración de AINES o aspirina, entre otros); por ello, es probable que existan otros factores que hayan contribuido en su aparición. La diabetes, la enfermedad diverticular y la metformina per se alteran el microbiota con la consiguiente disbiosis intestinal y síntomas digestivos molestos.

Freckelton et $a .^{10}$ realizaron una investigación observacional, reportaron una disminución de la incidencia de diverticulitis aguda, alrededor de $20 \%$ en diabéticos con enfermedad diverticular. Este efecto aparentemente benéfico de metformina estaría asociado a sus efectos antiinflamatorios. Por la naturaleza del estudio, los autores recomiendan que estos resultados se consideren con cautela y que se amplíen con estudios de intervención, randomizados y prospectivos para encontrar resultados significativos.
Se concluye que la aparición precoz de diarreas acuosas y otros síntomas gastrointestinales entre 24 a 72 horas de la administración de metformina debe plantear la sospecha de intolerancia a la misma, iniciando el manejo oportuno con una reducción de la dosis, metformina de liberación prolongada o insulinoterapia. Se sugiere no infravalorar la presencia de alteraciones anatómicas intestinales porque estas pueden contribuir significativamente en la fisiopatología de intolerancia a la metformina.

\section{Bibliografía}

1. Bouchoucha M, Uzzan B, Cohen R. Metformin and digestive disorders. Diabetes Metab. 2011; 37(2): 90-6. doi: 10.1016/j.diabet.2010.11.002.

2. Bonnet F, Scheen A. Understanding and overcoming metformin gastrointestinal intolerance. Diabetes Obes Metab. 2017; 19(4): 473-81. doi: 10.1111/ dom.12854.

3. Dujic T, Zhou K, Donnelly LA, Tavendale R, Palmer CNA, Pearson ER. Association of Organic Cation Transporter 1 With Intolerance to Metformin in Type 2 Diabetes: A GoDARTS Study. Diabetes. 2015; 64(5): 1786-93. doi: 10.2337/db14-1388.

4. Elbere I, Kalnina I, Silamikelis I, Konrade I, Zaharenko L, Sekace Ket al. Association of metformin administration with gut microbiome dysbiosis in healthy volunteers. PLoS One. 2018; 13(9): e0204317. doi: 10.1371/journal.pone.0204317.

5. McCreight LJ, Stage TB, Connelly P, Lonergan M, Nielsen F, Prehn C, et al. Pharmacokinetics of metformin in patients with gastrointestinal intolerance. Diabetes Obes Metab. 2018; 20(7): 1593-601. doi: 10.1111/dom.13264.

6. McCreight LJ, Bailey CJ, Pearson ER. Metformin and the gastrointestinal tract. Diabetología. 2016; 59: 426-35. doi: 10.1007/s00125-015-3844-9.

7. Zhou K, Donnelly LA, Kimber CH, Donnan P, Doney A, Leese G, et al. Reduced-function SLC22A1 polymorphisms encoding organic cation transporter 1 and glycemic response to metformin: a GoDARTS study. Diabetes. 2009; 58: 1434-1439. doi: 10.2337/db08-0896.

8. Wu H, Esteve E, Tremaroli V, Khan MT, Caesar R, Mannerås-Holm L, et al. Metformin alters the gut microbiome of individuals with treatment-naive type 2 diabetes, contributing to the therapeutic effects of the drug. Nat Med. 2017; 23(7): 850-8. doi: 10.1038/nm.4345.

9. Fluxá D, Quera R. Enfermedad diverticular: mitos y realidades [Diverticular disease: myths and realities]. Rev Med Chil. 2017; 145(2): 201-208. doi: 10.4067/S0034-98872017000200009.

10. Freckelton J, Evans JA, Croagh D, Moore GT. Metformin use in diabetics with diverticular disease is associated with reduced incidence of diverticulitis. Scand J Gastroenterol. 2017; 52(9): 969-72. doi: 10.1080/00365521.2017.1325930. 